\title{
The Research on Siting and Sizing of Electric Vehicle Charging Stations Based on Two Stage Optimization
}

\author{
Yu Jing ${ }^{a}$, Xueliang Huang ${ }^{b}$, Zhong Chen ${ }^{c}$ and Jun Cheng ${ }^{d}$ \\ School of Electrical Engineering, Southeast University, Nanjing 210096, China \\ abournejing@163.com, 'blhuang@seu.edu.cn, czhongchen@seu.edu.cn, \\ dchengjunseu@126.com
}

Keywords: Two stage optimization, electric vehicles, planning of charging station, cuckoo search algorithm.

\begin{abstract}
To meet the charging demand of the electric vehicles (EVs), a new two stage optimization (TSO) method is proposed in this paper with the consideration of the public service attributes of EV charging station (CS) and its impact on the distribution network. First, the locating and sizing scheme based on the multi-level capacity of CS is presented according to EV charging demand. Next, the optimal method of CS connected to the grid is derived from the point of view of the distribution network. Then, Mixed Integer Programming (MIP) and Improved Cuckoo Search (ICS) algorithm are sequentially applied to solve above optimization problem. Finally, experimental results manifest that the proposed model and algorithm are practical, useful and feasible.
\end{abstract}

\section{Introduction}

With the global energy crisis deepening and climate change problems growing over the past dacades, as a new type of energy saving and environment friendly transportation tool, electric vehicles (EVs) have become the focus of the global automotive industry for the future development and competition. Simultaneously, governments around the world have introduced policies to promote the development of EV industry. However, a comprehensive and sophisticated charging network is the premise and cornerstone for the promotion of EV industry. Therefore, theoretical study on the planning theory of the charging infrastructure is more important, especially in its infancy.

At present, the research on the siting and sizing of EV charging station (CS) has been carried out by scholars in China and abroad [1, 2]. In this paper, the EV CS, on one hand, is regarded as a public service facility for urban transportation, which can meet the needs of EV charging; on the other hand, EV CS transform the energy of the power grid into the battery carried with the EVs as the interface of the two part, making it possible to integrate large-scale EVs into the grid. With the rapid development of EVs, the effect of EV charging load and its characteristics on the distribution network cannot be ignored. Thus, the impact of EV charging stations accessing to the distribution network should be taken into account in the process of the layout of CSs. A new two stage optimization (TSO) method is proposed in this paper. First, the locating and sizing scheme based on the multi-level capacity of CS is presented according to EV charging demand. Next, the optimal method of CS connected to the grid is derived from the point of view of the distribution network.

In the next section, the location and capacity configuration of CSs are determined to minimize the investment and operation costs of CSs, which is based on the travelling needs in the first stage optimization from the perspective of the road network. Then, the model of the second stage optimization is described in Section 3 from the perspective of the power grid, which aims at the minimum network losses and fixed investment costs of connecting CSs to the grid according to the charging load for each CS and distribution network data. Section 4 highlights the Mixed Integer Programming (MIP) and the Improved Cuckoo Search (ICS) algorithm which are sequentially used to solve the TSO model proposed above. Section 5 demonstrates the applicability of the proposed model using a numerical example, while Section 6 offers the conclusions and directions for future research. 


\section{Model of the First Stage Optimization}

\subsection{EV Charging Demand Constraints.}

EV charging behavior is influenced by many factors, of which the most important is charging needs of owners [3]. As shown in Fig. 1, the EV charging demand constraints are illustrated through a simply example, supposing that an EV departs from the origin $O$, heading to the destination $D$ after points $i, j$ and $k$ respectively. Assuming that an EV $v$ can arrive at point $j$, the remaining energy at point $j$ for EV $v$ equals the sum of remaining energy and recharging energy at previous point $i$ minus the energy consumption between point $j$ and $i$; besides, the remaining energy at point $j$ for $\mathrm{EV} v$ should not be less than the SOC lower limit of the battery; at last, the sum of remaining energy and recharging energy at point $j$ for EV $v$ should not be more than the SOC upper limit of the battery.

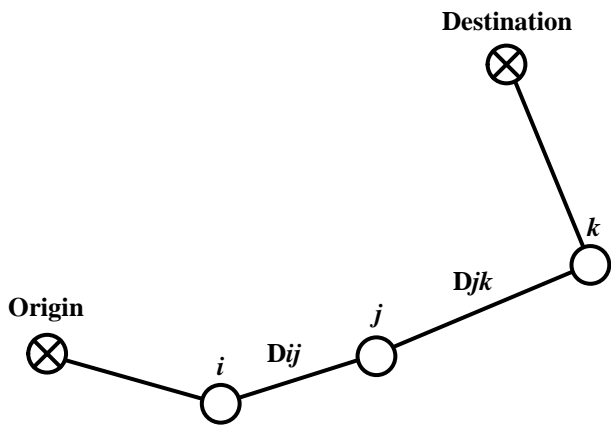

Fig. 1 The chart of EV charging demand constraints

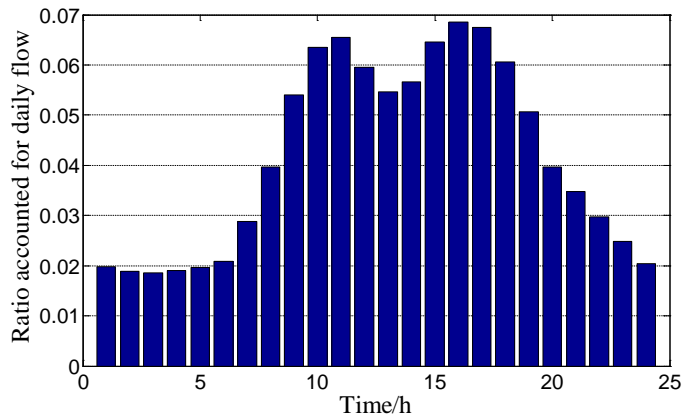

Fig. 2 The daily change curve of traffic flow

To be clear, the EV charging demand constraints can be formulated as the 3 following general expressions:

$B_{i v}+R_{i v}-D_{i j} \geq C \cdot S O C_{\min }$, where $B_{i v}$ is the remaining energy at point I for an EV $\mathrm{v}, R_{i v}$ is the recharging energy at point I for an $\mathrm{EV} \mathrm{v}, D_{i j}$ is the energy consumption between point $\mathrm{i}$ and $\mathrm{j}$, which is the equivalent of the distance between point $\mathrm{I}$ and $\mathrm{j}$; $\mathrm{C}$ is the battery capacity, $s \boldsymbol{c}_{\min }$ is the lower limit of the battery State Of Charge (SOC);

$B_{j v}=B_{i v}+R_{i v}-D_{i j}$, where $B_{j v}$ is the remaining energy at point $\mathrm{j}$ for an EV v;

$B_{i v}+R_{i v} \leq C \cdot S_{S O C_{\max }}$, where $S O c_{\max }$ is the upper limit of the battery (SOC).

\subsection{Model of the EV Flow.}

Based on the laws of Newtonian physics, the model elucidates that the EV flow is proportional to the attractiveness of the traffic zones and in inverse proportion to their distance.To generate flow on arc, all OD flows are allocated to shortest paths. The EV flow can be formulated as:

$$
f=L \frac{\omega_{i}^{\beta} \omega_{j}^{\beta}}{r_{i j}^{b}}
$$

where $f$ is the EV flow between point $\mathrm{i}$ and $\mathrm{j} ; \omega_{i}$ is the weight of the point $\mathrm{i} ; w_{j}$ is the weight of the point $\mathrm{j} ; r_{i j}$ is the distance of shortest path between point $\mathrm{i}$ and $\mathrm{j}$, which can be calculated through Dijkstra algorithm; $b$ is a coefficient which indicates the influence degree of spatial distance on gravity; $\beta$ reflects the relationship between the quantity of the EV users and the amount of the charging demand; $L$ is a constant for specific network model. The three above coefficients can be derived from the regression model based on previous data. Furthermore, it is worthy to note that the time unit of EV flow calculated by the above formula is one day.

\subsection{Optimum Siting and Sizing Model for EV CS.}

The needs of most EV users can't be meet through slow-charging mode when traveling, due to their sensitivity to charging time. On the contrary, the CSs in fast-charging mode can provide services in a short time with high energy density. Consequently, the fast-CSs become the main focus in this paper and 6 major assumptions are made as follows:

Candidate sites for CSs are the nodes of road network, that is to say, EV users only can accept services for charging at the nodes of road network;

All EV users are rational, which means they take the shortest path for each OD pair; 
Power consumption of an EV is linear with the distance of driving;

All EVs are the same type with the same battery capacity, namely identical maximum travel distance;

As the remaining capacity of an EV at origin point always maintain at a high level, it is assumed that $30 \%$ of those set out with $95 \%$ SOC, $40 \%$ of those set out with $90 \%$ SOC and the rest of those set out with $85 \%$ SOC;

In order to guarantee the service life of EV battery, overcharge should be avoided; similarly, the remaining energy of an EV shoud not less than a threshold in consideration of the concerns on travelling for EV users. So let $s o c_{\max }$ be $95 \%$ and $s o c_{\min }$ be $20 \%$.

The objective function is formulated as:

$$
\begin{aligned}
& \min \sum_{k \in \mathbf{V}}\left(f_{k, 1}+f_{k, 2}\right) \cdot X_{k} \\
& f_{k, 1}=\left(a X_{k}+b X_{k}+e_{k}\right) \cdot \frac{r_{0}\left(1+r_{0}\right)^{z}}{\left(1+r_{0}\right)^{z}-1} \\
& f_{k, 2}=\left(a X_{k}+b X_{k}+e_{k}\right) \cdot(1+\eta)
\end{aligned}
$$

Where $f_{k, 1}$ is the annual investment cost of CS $k, f_{k, 2}$ is the annual operation cost of CS $k, X_{k}$ is the capacity level of CS $k$, which is a nonnegative integer. $X_{k}$ is equal to 0 if there is no CS to be constructed at node $k$, otherwise $X_{k}$ is an integer; $a$ is the cost of transformer with unit capacity; $b$ is the cost of charger with unit capacity; $e_{k}$ is the cost of infrastructure construction for CS $k$, which is closely related to the location and the land rent; $r_{0}$ is the lending rate; $z$ is the operational life; $\eta$ is the conversion factor; the operation cost of CS can be expressed as a function involved with initial investment.

The constraints are as follows:

EV charging demand

$$
\begin{aligned}
& B_{k v} \geq C \cdot S O c_{\text {min }} \quad \forall k \in \mathbf{N}, \forall v \in \mathbf{V} \\
& B_{k v}=B_{j v}+R_{j v}-\sigma_{j k v} \times D_{j k} \forall j k \in \mathbf{A}, \forall v \in \mathbf{V} \\
& B_{k v}+R_{k v} \leq C \cdot S O c_{\text {max }} \quad \forall k \in \mathbf{N}, \forall v \in \mathbf{V}
\end{aligned}
$$

Where $\boldsymbol{N}$ is the set of nodes in the road network; $\boldsymbol{A}$ is the set of arcs in the road network; $\boldsymbol{V}$ is the set of EVs; $\sigma_{j k v}$ is a binary variable. $\sigma_{j k v}$ is equal to 1 if an EV $v$ passes by arc $j k$, otherwise $\sigma_{j k v}$ is 0 .

EV CS capacity constraint

$$
\sum_{v \in \mathbf{V}} R_{k v} \cdot \alpha_{v} \leq \gamma \cdot U \cdot X_{k} \forall k \in \mathbf{N}
$$

Where $\alpha_{v}$ is a flow coefficient for EV $v$, which can be obtained from the gravity model; $\gamma$ is a factor stands for the driving distance per $k W h ; U$ is capacity unit of CS.

Other constraints

$$
\begin{aligned}
& R_{k v} \geq 0 \quad \forall k \in \mathbf{N}, \forall v \in \mathbf{V} \\
& 0 \leq X_{k} \leq X_{\text {max }} X_{k} \in \text { \{nonnegative integers }
\end{aligned}
$$

Where, $X_{\max }$ is the maximum capacity level of CS.

Note that, the outputs of the model above are the sum of charging demand in one day for each CS. For this reason, the capacity configuration for each CS can be determined based on the following conversion formula.

After analysing and processing the data from the road netwok in Shandong province [4], the daily change curve of traffic flow can be fitted as Fig. 2. It is reasonable to assume that EV flows in the study area obey the trend shown below and there is a linear relationship between charging demand and EV flow. Then, the hourly charging loads could be evaluated. Finally, the capacity configuration can be expressed as the peak charging load multiplied by a margin factor, as equation (11) shows.

$$
\operatorname{Cap}_{k}=\max _{t}\left\{P_{k}(t)\right\} \times \Phi
$$

Where $\operatorname{Cap}_{k}$ is the capacity of CS $k ; P_{k}(t)$ is the active power of CS $k$ at time $t$; $\Phi$ is a margin factor. 


\section{Model of the Second Stage Optimization}

After optimum siting and sizing of CSs based on the travelling needs in the first stage optimization from the perspective of the road network, the impact of EV CSs accessing to the distribution network is taken into account in the second stage optimization. An optimal method of CS connected to the grid is presented, which intends to minimize the network losses and fixed investment costs of connecting CSs to the grid.

\subsection{Objective Function.}

$$
\begin{aligned}
& \min \lambda_{1} c_{1}+\lambda_{2} c_{2} \\
& c_{1}=\sum_{i=1}^{m} \sum_{k=1}^{h} \delta_{i k} \mu \sqrt{\left(u_{i}-x_{k}\right)^{2}+\left(v_{i}-y_{k}\right)^{2}} \\
& \delta_{i k}=0 \text { or } 1 \\
& C_{2}=\sum_{t=1}^{24} P_{\text {loss }, t}\left(\delta_{i k}, P_{k}(t), Q_{k}(t)\right)
\end{aligned}
$$

Where $c_{1}$ is the investment cost of new lines; $c_{2}$ is the network losses; $\lambda_{1}, \lambda_{2}$ is the weight coefficient of the above objective respectively; $\delta_{i k}$ means the connectivity of the node $i$ in distribution network and the node $k$ in road network. $\delta_{i k}$ is equal to 1 if the node $i$ in distribution network is connected to the node $k$ in road network, otherwise $\delta_{i k}$ is zero; $\mu$ represents the investment cost per kilometer; $\left(u_{i}, v_{i}\right)$ is the coordinate of node $i$ in distribution network; $\left(x_{k}, y_{k}\right)$ is the coordinate of node $k$ in road network; $P_{\text {loss, } t}(\cdot)$ is the network loss function at time $t$ which involves $\delta_{i k}, P_{k}(t)$ and $Q_{k}(t)$. $Q_{k}(t)$ is the reactive power of CS $k$ at time $t$.

\subsection{Constraints.}

Connecting constraint

$$
\sum_{i=1}^{m} \delta_{i k}=1 \quad k=1, \ldots, \mathrm{h}
$$

Branch power flow constraint

$$
P_{l} \leq P_{l_{\max }} l \in L
$$

Where $P_{l}$ is the active power of branch $l ; P_{\text {Imax }}$ is the maximum active power of branch $l$; $L$ is the set of branches.

Power balance constraint

$$
\left\{\begin{array}{c}
-P_{D i}(t)-\delta_{i k} P_{k}(t)-U_{i}(t) \sum_{j=1}^{n} U_{j}(t)\left(G_{i j} \cos \theta_{i j}(t)\right. \\
\left.+B_{i j} \sin \theta_{i j}(t)\right)=0 \\
-Q_{D i}(t)-\delta_{i k} Q_{k}(t)-U_{i}(t) \sum_{j=1}^{n} U_{j}(t)\left(G_{i j} \sin \theta_{i j}(t)\right. \\
\left.-B_{i j} \cos \theta_{i j}(t)\right)=0
\end{array}\right.
$$

where $P_{D i}, Q_{D i}$ is the active and reactive power of node $i$ in distribution network separately; $U_{i}, U_{j}$ is the voltage amplitude of node $i$ and $j ; \theta_{i} 、 \theta_{j}$ is the voltage phase angle of node $i$ and $j$; $\theta_{i j}$ represents $\theta_{i}$ minus $\theta_{j}$; $G_{i j}$, $B_{i j}$ is the real and imaginary part of the element of node admittance matrix at row $i$ and column $j$.

Node voltage constraint

$U_{i, \text { min }}(t) \leq U_{i}(t) \leq U_{i, \max }(t)$

Where $U_{i, \max }, U_{i, \min }$ is the upper and lower limit of voltage amplitude of node $i$. 


\section{Solution to Solve TSO Model}

To solve the TSO model proposed above, the Mixed Integer Programming (MIP) and the Improved Cuckoo Search (ICS) algorithm are applied sequentially.

\subsection{Mixed Integer Programming.}

In mathematical sense, the model of first stage optimization belongs to set covering problem and could be solved exactly by branch and bound. In this paper, the IBM's business mathematical programming optimization program CPLEX is exploited to solve the first stage optimization.

\subsection{Improved Cuckoo Search Algorithm.}

Due to the nonlinear, multi-dimensional and non-convex characteristic manifested in the second stage optimization, an ICS algorithm with a strong ability to reach the global optimum region is presented [5]. In the basic cuckoo search, new solutions can be generated by equation (19). $\alpha$ is set to 1 as a constant step size. However, the lack of automatic correction for search step size leads to inaccuracy during the final optimization phase. As a result, an ICS algorithm with adaptive step size can quicken convergence towards the optimality in the whole search.

$$
\begin{aligned}
x_{i}^{(\mathrm{G}+1)} & =x_{i}^{(\mathrm{G})}+\alpha \cdot \operatorname{Lévy}(\lambda) \quad i=1,2, \cdots, \mathrm{N} \\
\alpha & =e^{-30 \times\left(t / T_{\max }\right)^{s}}
\end{aligned}
$$

Where $t$ is the current iteration time, $T_{\max }$ is the maximum iteration time; $s$ is an attenuation coefficient which ranges from 1 to 30; Lévy $(\lambda)$ represents a random variable drawn from a Lévy distribution with $\lambda$ parameter.

Note that the second stage optimization model has several constraints. This programming problem should be converted to an unconstrained problem through penalty function method in this article.

\section{Case Study}

In this section, the proposed model and solution is implemented in a typical area, where a 33 nodes distribution system [6] as shown in Fig. 3 and a 25 nodes road network system [7] as depicted in Fig. 4 have been built.

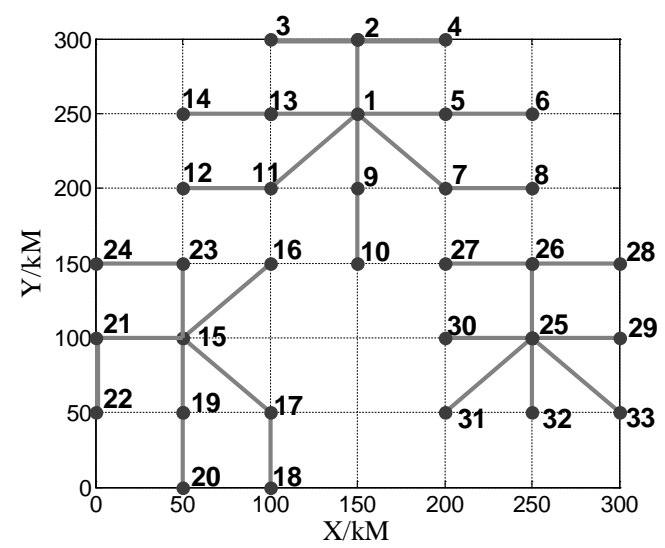

Fig. 333 nodes distribution system

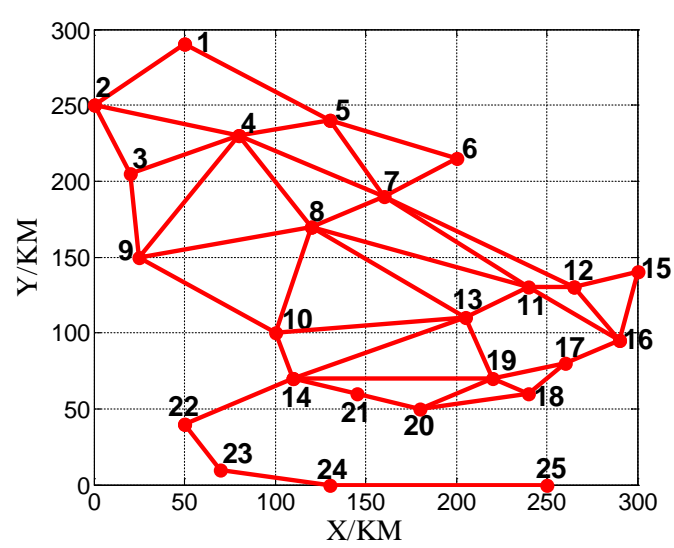

Fig. 425 nodes road network system

In the first stage optimization, let $\gamma$ be $5.13 \mathrm{kM} / \mathrm{kWh}$ and $C$ be $150 \mathrm{kM}$ according to BYD E6 and let the power factor of chager be $0.9 ; a, b$ and $e_{k}$ is taken as 100 thousend yuan, 100 thousend yuan and 200 thousend yuan respectively; Assume $r_{0}$ is $7 \%, z$ is 5 year, $\eta$ is $-0.6, X_{\max }$ is $3, U$ is $10000 \mathrm{kWh}$ and $\Phi$ is 1.2 ; In the gravity model, $L$ is $3, \beta$ is 1 and $b$ is 2 ; in the second stage optimization, $\lambda_{1}$ is set to 0.4 , $\lambda_{2}$ is set to 0.6 and $\mu$ is 20 thousend yuan; $N$ is 25 and $P_{a}$ is 0.25 .

After the first stage optimization, the optimum location and capacity configuration of CSs are derived, which are shown in Fig. 5. The chart presents that there is lower charging demand at the 
nodes such as node 1, 2 and 6 and this amount of demand can be shift from node 1, 2 and 6 to their nearby nodes. For some busy and important nodes in road network, the possible charging demand pressure may bring about a higher capacity level. For instance, a 2.46MW CS need to built at node 22 to satisfy the charing demand.

After the second stage optimization, the optimal method of CS connected to the grid is described in Fig.6. According to this figure, the red rectangles are CSs achieved from the previous optimization; the grey points and lines are the nodes and branched in distribution network; the red lines represent the new branches to be constructed. It appears that with the consideration of network loss and investment cost some CSs are connected to closer nodes in distribution network like CS 3; some are connected to farther nodes, which may increases the investment cost but reduces the network loss greatly.
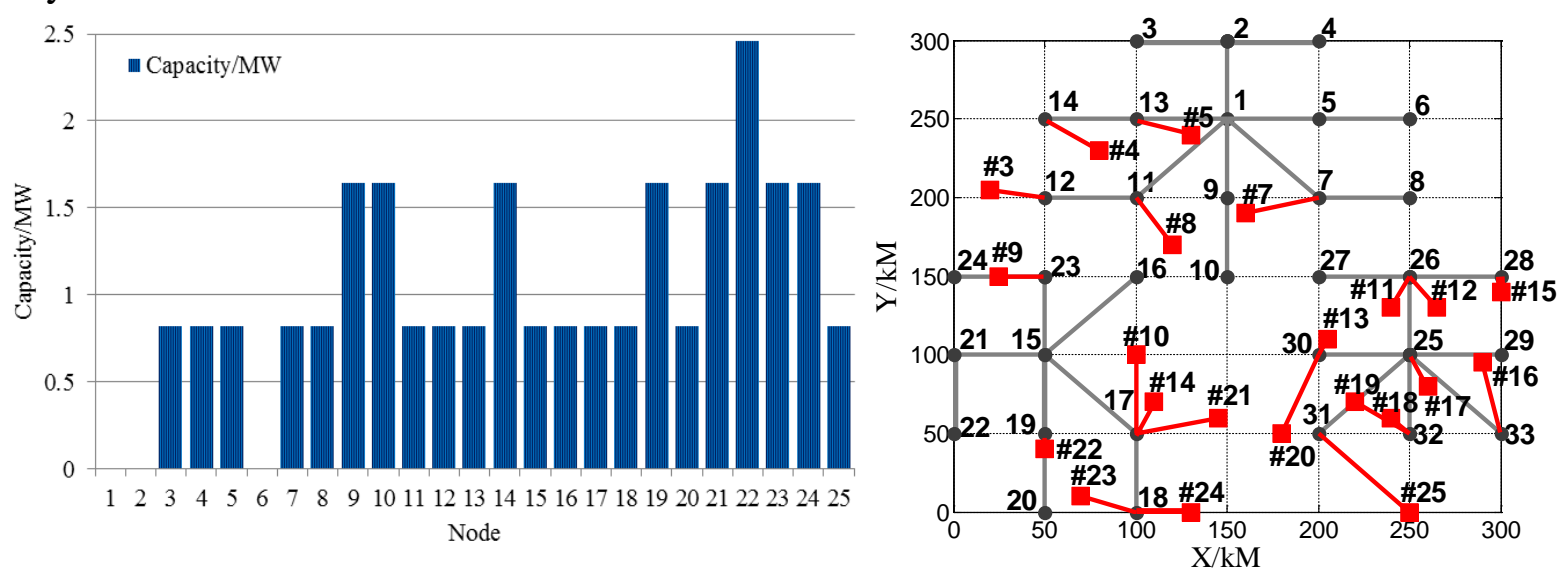

Fig. 5 Capacity configuration of CSs

Fig. 6 Method of CS connected to the grid

\section{Summary}

In this paper, a new TSO method is presented with the consideration of the public service attributes of EV CS and its impact on the distribution network. Specifically, a locating and sizing scheme and an optimal method of CS connected to the grid are proposed from the perspective of the road network and the distribution network respectively. Then, MIP and ICS algorithm are sequentially applied to solve above optimization problem. Finally, a typical case is simulated to test and verify the model and algorithm.

For complex CS layout planning, proper simplifications are made in this article. However, the model and the algorithm are sustainable and expansible. Further efforts should be make to determine optimal parameter values for the model and consider more actual conditions in the future research.

\section{Acknowledgments}

This work was supported by State Grid Corporation of China.

\section{References}

[1]. Kuby M, Lim S. The Flow-Refueling Location Problem for Alternative-Fuel Vehicles [J]. Socio-Economic Planning Sciences, 2005, 39(2): 125-145.

[2]. T. Donna Chen, Kara M. Kockelman, Moby Khan. The Electric Vehicle Charging Station Location Problem: A Paring-based Assignment Method for Seattle [J]. HKSTS 2012: Transportation and Logistics Management, p317-324, 2012.

[3]. Wang Y W, Lin C C. Locating Road-Vehicle Refueling Stations [J]. Transportation Research Part E: Logistics and Transportation Review, 2009, 45(5): 821-829.

[4]. Yu X H. Temporal and Spatial Analysis on Freeway Traffic Flow [D]. Shandong University of Science and Technology, China, 2009. 
[5]. Yang X S, Deb S. Cuckoo Search via Lévy Flights[C]//Nature \& Biologically Inspired Computing, 2009. NaBIC 2009. World Congress on. IEEE, 2009: 210-214.

[6]. GE S Y, FEND Liang, LIU Hong, et al. Planning of Charging Stations Considering Traffic Flow and Capacity Constraints of distribution network [J]. Power System Technology, 2013, 37(3): 582-589.

[7]. Hodgson M J. A Flow-Capturing Location-Allocation Model [J]. Geographical Analysis, 1990, 22(3): 270-279. 